\title{
A real time power line detection system based on stereo vision
}

\author{
Xingang MOU ${ }^{1, a}$, Wei LUO ${ }^{2, b}$, Xiaoliang $\mathrm{ZHENG}^{1, \mathrm{c}^{*}}$ and \\ Xiao $\mathrm{ZHOU}{ }^{1, d}$ \\ ${ }^{1}$ School of Mechanical and Electronic Engineering, Wuhan University of Technology, Wuhan, \\ China \\ ${ }^{2}$ Ewatt Technology Co,.Ltd, Wuhan, China \\ amouxingang@163.com, b25381917@qq.com , ’jayash@qq.com , d17895099@qq.com
}

Keywords: power line, FPGA, real time, stereo vision, 3d reconstruction

Abstract:In recent years, Unmanned Aerial Vehicle(UAV) is widely used for power lines patrol. While it's more efficient than manually patrolling, the UAV may easily collide with power lines. So measures must be taken to detect the distance between UAV and power lines. This paper presents a real time power line detect system based on binocular vision techniques, which contains a hardware platform based on FPGA and DSP to calculate real time disparity map and extract power lines. We adopt a robust stereo matching algorithm based on cross-based arbitrary shape support region and Hough transfer to extract lines from the depth image. Our experiment results show that our system can correctly detect the distance between the UAV and power lines in real time.

\section{Introduction}

Power lines patrol is a very important part for the security and stability of a power system. At present, this work is mainly completed manually, which is low efficiency and wasteful of labor. In recent years, Unmanned Aerial Vehicle(UAV) is widely used in more and more scenarios with the development of technology, for instance, aerial photograph, reconnaissance, safety inspection and express, etc. However, serious hazards exist when aerial vehicles operated at low altitude in cluttered environments without constrained or controlled conditions of different obstacles, lighting effects, weather and so on. Specially, power lines are one of the most formidable hazards [1]. Therefore, obstacle avoiding is the critical technology of UAV.

Visual image technology has more broad detection range, higher accuracy than sonar technology and cost less than radar technology. With the decline cost and the improved computing ability of mobile chip, visual image technology becomes the first choice to achieve obstacle avoiding. The use of binocular vision technology to avoid obstacles in the UAV is a hot research topic in recent years. The character of real-time of binocular stereo vision algorithm becomes the fundamental reference index due to its complication. In recent years, more and more binocular vision system based on FPGA achieved real-time disparity maps with good results [2].

This paper presents a real time power line patrol system based on FPGA, which contains a real-time disparity map and image characteristic of power line extraction algorithm. This system can calculate the distance between the closest line and the UAV in real time, according to the relevant parameter and the real-time disparity map. 


\section{Related works}

\section{Binocular Vision}

Binocular vision techniques are widely used in computer vision for $3 \mathrm{D}$ reconstruction. The stereo system can infer the depth information by means of triangulation. The first aim is to find the corresponding points in two images captured by two cameras. The stereo system is showed as follows:

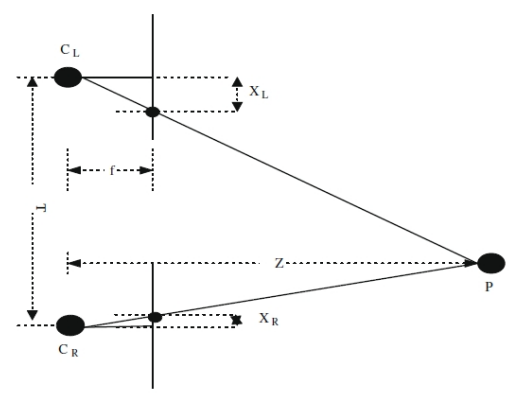

Fig. 1 geometric relationships of stereo system

In Fig. 1, CL and CR represent the optical centers of the left and right cameras, respectively. $\mathrm{T}$ is the distance between the two cameras (which is called baseline) and $\mathrm{f}$ is the focal length of the camera. The disparity of the point $\mathrm{P}$ is given by:

$$
d=X_{l}-X_{r}
$$

Reconstruction involves determining the three-dimensional scene using the disparity of points that correspond in both images, based on known camera geometry. In Fig. 1, the depth of the point $\mathrm{P}$ from the two cameras is given by

$$
d=f * \frac{T}{d}
$$

\section{Stereo Matching Algorithm and hardware Implementation}

A lot of algorithms have been proposed in recent years. Currently there are two kinds of stereo matching algorithms: one is local methods, which compute depth at a local support region. The other is global methods, which compute depth based on a global cost optimization. While global methods can achieve a more accuracy depth map than local ones, it also brings huge pressures on hardware resources due to complex computational method. For this reason, most hardware solutions select local methods as the stereo matching algorithm.

GPU and FPGA are two main hardware platforms used to accelerate stereo matching. While it is much easier to transplant an existed algorithm into GPU, a FPGA based system can achieve a more flexible configuration and usually has lower power consumption.

Zhang et al. [4] used a dsp-bulider based FPGA system to implement stereo matching, achieving $30 \mathrm{f} / \mathrm{ps}$ at resolution of $1396^{*} 1110$ with 128 disparity levels. Wang et al. [3] transplant AD-Census algorithm into FPGA with semi-global optimization after cost aggregation, which is able to process high definition images of $1600 * 1200$ pixels at 42 frames per second. Abdulkadir et al. [5] proposed a new algorithm named AWDE, which is very suitable to be implement on hardware, they implemented their algorithm on Xilinx Vitex-5 and can achieve $60 \mathrm{f} / \mathrm{ps}$ at resolution of $1024 * 768$ with 128 disparity levels. Zhang et al. [6] combine the mini-census transform and cross-based cost aggregation in their structure, which achieves 60 frames/s at $1024 \times 768$ pixel stereo images. 


\section{System Overview}

The aim of our work is to extract power lines in real time. A binocular vision system must be provided in our system. Considering that the system use FPGA to calculate depth image, the binocular cameras must have an interface that is easy to control. We select camera-link cameras in our system. The cameras connect to the FPGA by LVDS interface with a usart configuration interface.

Before we put the input video stream into the stereo module, we must correct the input images to compensate the distortion of the cameras, which we call camera calibration. Offline calibration is done with the help of MatLab calibration toolbox.

Then the corrected reference image streams and target image streams are connected to the stereo modules to get a real time depth image, which we will describe in detail in the next section.

After we get the real time depth map, measures must be taken to extract power lines from the depth map. Considering that the line extract algorithms will cost considerable hardware resources, a better solution is using a DSP to complete line extract algorithms. A dual port ram is used for the DSP and FPGA to communicate with each other. Also, the dpram stores the line extract result and send it to the video output controller to generate output video stream. The overall structure of the system is as follows:

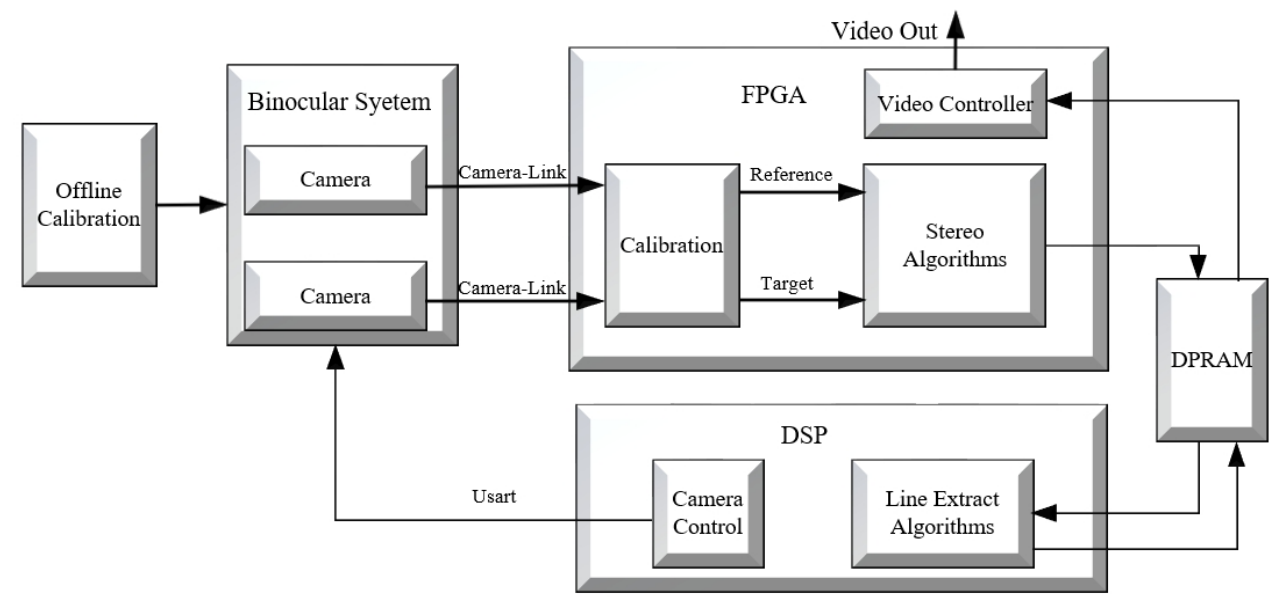

Fig. 2 overall structure of the detect system

\section{Algorithm flow}

\section{Stereo matching algorithm}

An accurate depth map should be obtained before detecting the power lines from the captured images. In this case, stereo matching algorithm is the most important part of this paper. In this section, we discuss the algorithm selection for stereo matching. Firstly, we give the overall algorithms structure as follows:

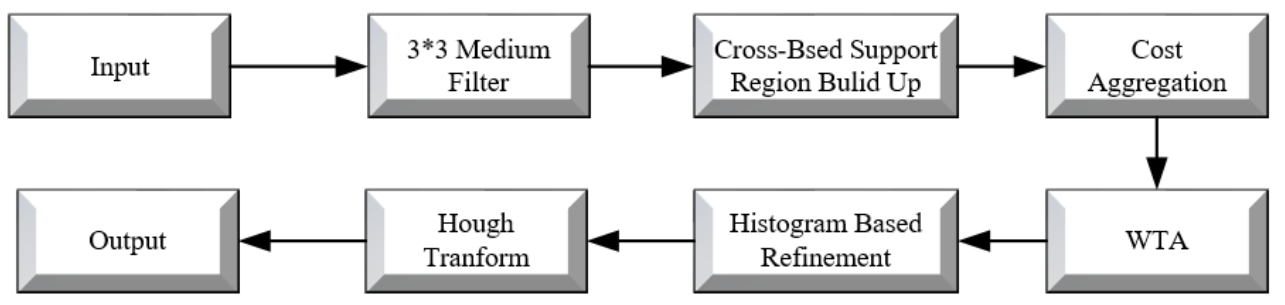

Fig. 3 overall structure of the algorithm 
According to the taxonomy proposed in [7] most stereo algorithms perform these steps:

1) Pre-processing

For stereo matching, either support region build-up task or cost aggregation task can easily be disturbed by noises from the image sensors. In this paper, a medium filter with filter window size $3 * 3$ will be used as preprocessing method.

2) Matching cost computation

Matching cost computation is the method to decide the correspondence of the target pixels in the reference image and the target image. Absolute Difference(AD), Birchfield and Tomasi's sampling-insensitive measure(BT) gradient-based measures and Census based measures are frequently used in stereo matching. While census is proved to be insensitive to radiometric distortion, it brings extra computation efforts and also introduces matching ambiguities in image regions with repetitive or similar local structures [8]. In fact, the AD measures behave better in our system, so we select $\mathrm{AD}$ as the matching cost computation. The definition of the AD cost is as follows:

$$
C_{A D}=\frac{1}{3} \sum_{i=R, G, B}\left|L_{i}^{\text {Left }}(p)-L_{i}^{\text {Right }}(p, d)\right|
$$

3) Cost aggregation

Stereo matching work is done by finding the correspondence relationship of the pixels between the target image and the reference image. However, single pixel comparison is easily affected by noises or ambiguities. So a cost aggregation step is done on a series of pixels which we call support regions. Fixed rectangle support window ignores depth discontinuities and is not suited for accurate occasions. For our power line detect mission, power line images contain a lot of discontinuous depth information and the line depth information is easy to be swallowed up in background pixels if fixed window region is adopted.

Zhang et al. [9] proposed a cross-based region build-up method which can achieve much better depth for depth discontinuities.

The cross-based method firstly calculates four arm point for the anchor pixel $\mathrm{p}$ accord to the color similarity, which is defined as follows:

$$
\sigma\left(p_{1}, p_{2}\right)=\left\{\begin{array}{l}
1, \max \left(I c\left(p_{1}\right)-I c(p 2)\right) \leq \tau \\
0, \text { otherwise }
\end{array}\right.
$$

The corresponding arm length is defined by:

$$
r^{*}=\max _{r \in[1, L]}\left(r \prod_{i \in[1, r]} \delta\left(p, p_{i}\right)\right)
$$

Then a cross based region is built up by scanning from the top to bottom to calculate every horizontal arms of $q$ of the vertical arm which p belongs to.

The cost aggregation task is done on the overlapping region of the reference image and target image. To normalize the cost aggregation result, the total pixels in each support region must be recorded previously.

4) Depth Select

For most local stereo matching method, we take a Winner Takes All (WTA)method to get the preliminary estimated value. WTA method takes the minimum value of the aggregate result as the estimated value, which is:

$$
d_{p}^{0}=\arg \left(\min _{d} \overline{\mathrm{E}_{\mathrm{d}}}(\mathrm{p})\right), \mathrm{d} \in\left[0, d_{\max }\right]
$$


5) Disparity refinement

The preliminary estimated value can be further refined using a local high-confidence voting scheme proposed in [10]. the final disparity of the pixel $p$ is decided as:

$$
d_{p}^{*}=\arg \left(\max _{d} \varphi_{p}(\mathrm{~d})\right), \mathrm{d} \in\left[0, d_{\max }\right]
$$

Where $\varphi_{p}$ is the histogram of the initial disparity estimate $d_{s}^{0}$.

\section{Line Detecting Algorithm}

After we get an accurate depth map, the next step is to extract lines from the depth map. Here we use Hough Transform to do this job. Hough transform is an effective algorithm to detect straight lines from the image, which is the main feature of the power lines.

\section{FPGA Implementation}

The FPGA system include 4 sub modules:

1) Video capture module

This module captures video from camera link cameras. Firstly, FPGA buffers the LVDS RGB data using a lvds buffer; then the video data is pushed into a cross clock fifo to sync to local clock domain. Then the video streams are calibrated by calibration circuits to compensate for camera distortion.

2) Stereo Matching module

This module performs the stereo matching using a full parallel structure which is showed as Figure 5 . Firstly, a $3 * 3$ medium filter will be performed.

3) DPRAM controller module

This module contains two sub circuits. One is write module to write depth results into dpram. The other is read module to read line segment result processed by DSP from the dpram.

4) Video Controller module

This module generates output video timing.

The overall structure of the FPGA system is showed as figure 4.

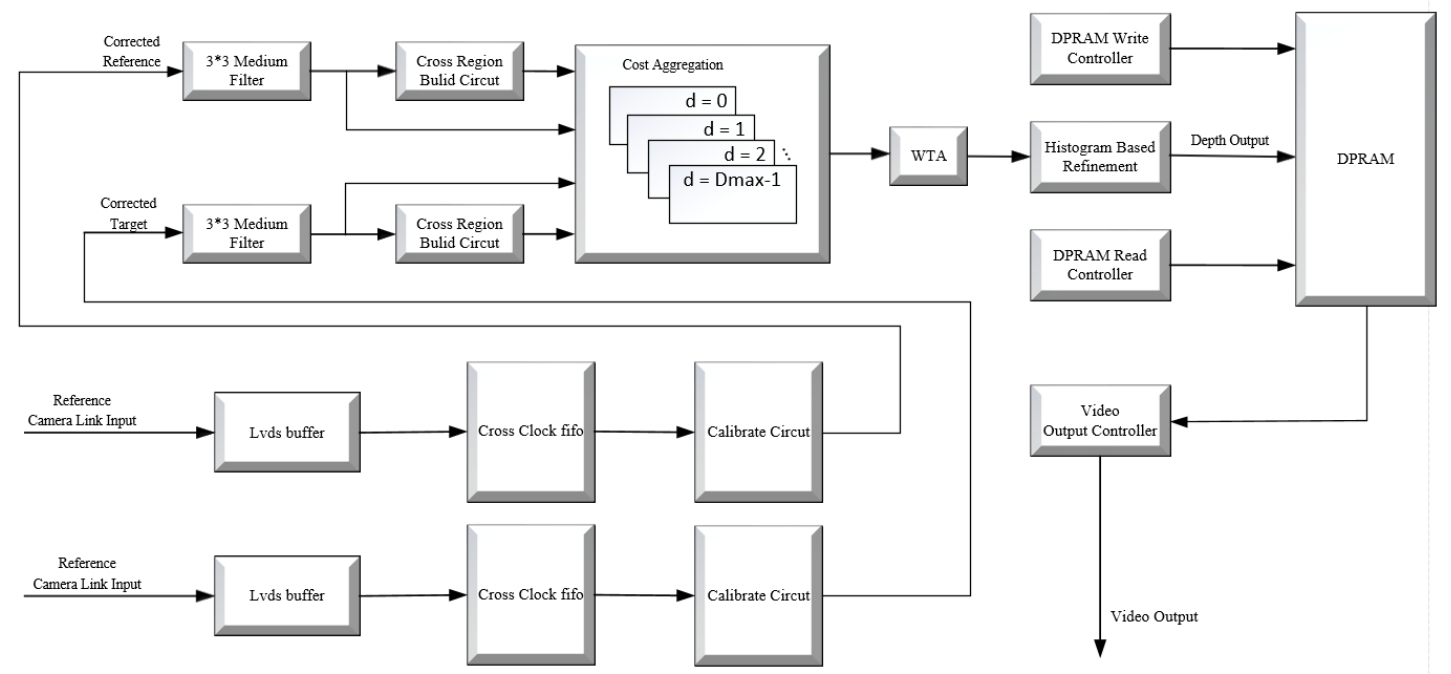

Figure 4. The overall structure of the FPGA system 


\section{Experimental Results}

Firstly, we test the algorithms with the Middlebury benchmark [10]. The disparity results are presented in Figure 5.
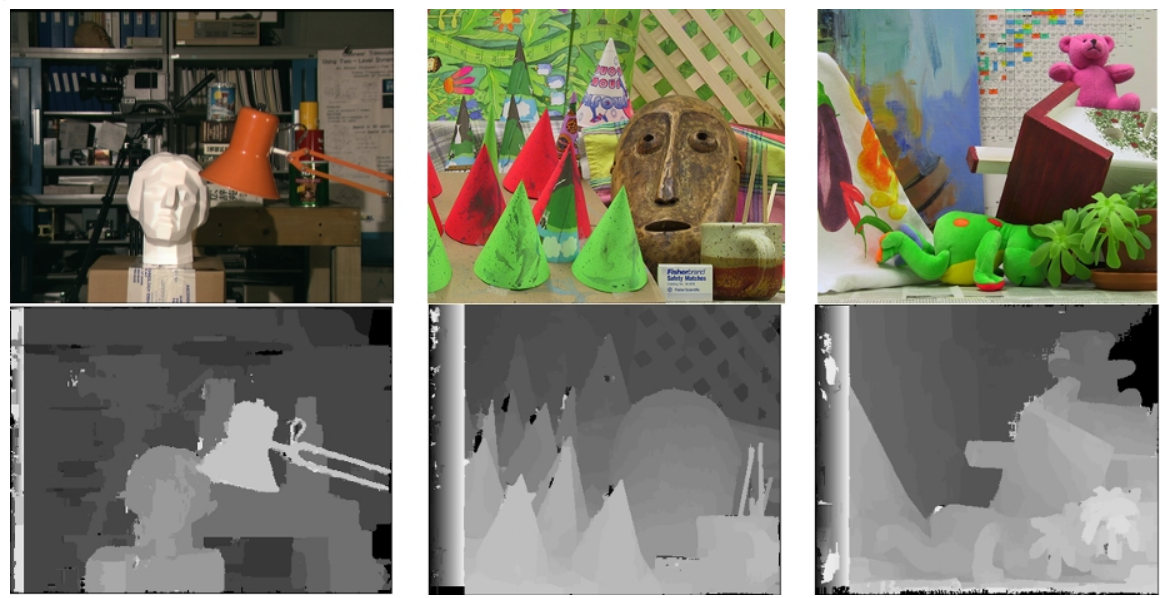

Fig. 5. Final disparity maps for the Tsukuba, Cones and Teddy stereo datasets (from left to right), using cross-based stereo matching algorithm. Top row: the input left images. Bottom row: the resulting disparity maps.

Then we test the real world power line images as figure 6 .
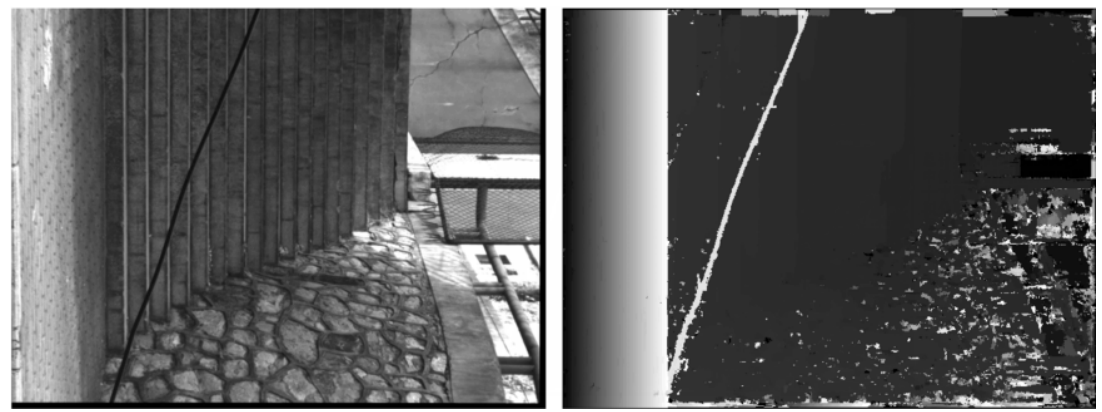

Fig. 6. Final disparity maps for the real world power line image, the left image: power line image; the right image: the resulting disparity maps.

\section{Conclusion}

In this paper, we propose a real time hardware platform to detect power lines for the UAV power line patrol system. The experiment results show that the stereo matching algorithms are robust and can get accurate depth information from the vision system. Power lines can be extracted from the depth images correctly.

\section{References}

[1] Song B, Li X. Power line detection from optical images[J]. Neurocomputing, 2014, 129(129):350-361.

[2] Wang, Wenqiang, et al. "Real-time high-quality stereo vision system in FPGA." International Conference on Field-Programmable Technology IEEE, 2013:358-361.

[3] Simhadri V, Ozturk Y. RASCor: An associative hardware algorithm for real time stereo[J]. Computers \& Electrical Engineering, 2009, 35(3):459-477.

[4] Zhang X, Chen Z W. A FPGA Stereo Matching Algorithm Modeled By DSP Builder[J]. Journal of Computers, 2014, 9(10).

[5] Akin A, Baz I, Schmid A, et al. Dynamically adaptive real-time disparity estimation hardware 
using iterative refinement[J]. Integration the Vlsi Journal, 2013, 47(3):365-376.

[6] L. Zhang, K. Zhang, T. S. Chang, G. Lafruit, G. K. Kuzmanov, and D. Verkest, "Real-time high-definition stereo matching on FPGA," in Proc. 19th ACM/SIGDA Int. Symp. Field Program. Gate Arrays, 2011, pp. 55-64.

[7] D. Scharstein and R. Szeliski, A taxonomy and evaluation of dense two-frame stereo correspondence algorithms Int. Jour. Computer Vision, 47(1/2/3):7-42, 2002

[8] Mei, Xing, et al. "On building an accurate stereo matching system on graphics hardware." IEEE International Conference on Computer Vision Workshops, ICCV 2011 Workshops, Barcelona, Spain, November 2011:467-474.

[9] Zhang K, Lu J, Lafruit G. Cross-based local stereo matching using orthogonal integral images[J]. IEEE Transactions on Circuits \& Systems for Video Technology, 2009, 19(7):1073-1079.

[10] D. Scharstein and R. Szeliski. Middlebury stereo evaluation -version 2, 2010.

http://vision.middlebury.edu/stereo/eval/. 